doch gar nicht machen, als durch Nachprüfung der in beiden Fällen erzielten Wirkungen. Auch hier liegt der vielfach gemachte Grundfehler vor, das auf seine Neuheit nachzuprüfende Verfahren gewissermaken auf einen schlagwortartigen Begriff zuzuspitzen und nun bei Entdeckung des gleichen Ausdruckes in einer früheren Veröffentlichung kritiklos die Erfindung zu verneinen. Die wirkliche Entwicklung des Rigbyschen Verfahrens (vgl. The Iron and (Coal Trades Review vom 23./9. 1910) beweist anch weiter, daß die der Patentschrift Rigby unterschobenen Entwicklungsmöglichkeiten in Wirklichkeit nicht bestanden haben, denn der Imweg über die Jauge ist immernoch nicht beseitigt.

Ferner weist $\mathrm{B}$ a $\mathrm{g}$ l e y darauf hin, aus dem britischen Patent 20 870/1904 ginge hervor, da $\mathrm{K}$ o p pers sich selbst ïber sein Verfahren damals noch nicht klar gewesen wäre, lemn in dieser Patentschrift hïtte er ein heißes Verfahren beschrieben. Ich muß hier darauf hinwejsen, daß diese Behauptung jedenfalls nur auf (Grund eines oberflächlichen Studiums dieser Patentschrift aufgestellt werden konnte: Dem aufmerksamen Leser kann es nicht entgehen, daß in der britischen Patentschrift 20 870/1904 die ganze Entwicklung des einschlägigen Gebietes enthalten ist, indem nebeneinander das heiße und das kalte Verfahren besehrieben sind, wobei die Vorteile des kalten Verfahrens schon damals vollständig gewürdigt wurden. Es kann für denjenigen, der einmal die Geschichte der direkten Verfahren schreiben will, nicht genug empfohlen werden, gerade dieser Patentschrift seine besondere Aufmerksamkeit zu widmen!

Den größten Teil seiner Ausführungen widmet $B$ a g 1 e y dem neuen Still'schen Verfahren, und er versucht, gegenüber dem deutschen Patent 181846 grundlegende Unterschiede zu konstruieren, indem er bis in die Einzelheiten einen Ver gleich durchführt. Ich brauche hier bloß nach wie vor hochzuhalten, daß für jeden unbefangenen Betrachter eine grundsätzliche Ubereinstimmung der beiden Verfahren unverkennbar ist. Still hat lediglich den indirekten Wärmeaustausch zwischen Rohgas und Kondensat, der bei dem Koppers'schen Ausführungsbeispiel vorgesehen ist, durch einen direkten ersetzt, was aber nicht von grundsätzlicher Bedeutung ist, besonders deshalb nicht, weil ja ein derartiger direkter Wärmeaustausch beispielsweise durch das Mondsche Verfahren in großem Umfange bekannt geworden ist. Was ich mit meinen Ausführungen beweisen wollte, ist die Tatsache, daß jedenfalls der Grundgedanke: unter Ausnutzung der in dem Gase steckenden Wärme die ausgefallenen Wassermengen wieder in das Gas zurückzuführen, bereits von Ko p p e r s bekannt gegeben ist. Schon die Tatsache, daß nebeneinander die Firmen Otto und Still die Sache gleichzeitig in die Praxis gesetzt haben, und der zwischen den beiden Firmen getätigte Vergleich läßt doch bezüglich der Erfindung Schlüsse zu, deren Berechtigung nicht von der Hand zu weisen ist. Nur auf einen Punkt brauche ich hier hinzuweisen: B a g l e y stellt den in dem Still'schen Verfahren enthaltenen Unterschied des direkten Wärmeaustausches als einen ungeheuren Vorteil hin, auf der anderen Seite betont er (vgl. S. 382), daß ,die erste Patentanmeldung von der Firma Dr. C. Otto \& Co. auf ein in der Grundidee mit dem Stillschen Verfahren wesentlich übereinstimmendes Verfahren" eingereicht worden sei. Nun kann dieser eigenen Bekundung von $S t i l l$ gegenüber bloß darauf hingewiesen werden, daß gerade diese Patentanmeldung von $\mathrm{O}$ t to (vgl. die Anmeldung 7671, O. Kl. 26d vom 10./7. 1911 und das dieser entsprechende britische Patent 15584/1912) ausgerechnet den indirekten Wärmeaustausch enthält, den Still Koppers gegenüber als den grundsätzlichen Unterschied seines Verfahrens ansieht. Ein Kommentar hierzu dürfte sich wohl erübrigen!

Wenn B a gle y darauf hinweist, daß auch Still ein englisches und ein amerikanisches Patent erhalten habe, so beweist diese Tatsache für die Erfindung gar nichts: Ohne hier der Prüfung zum Patent angemeldeter Erfindungen in Amerika und England nahe treten zu wollen, will ich hier bloß hervorheben, daß in beiden Ländern das überaus wichtige Einspruchverfahren, wie es in Deutschland Gesetz ist, nicht besteht. Ich habe schon in einem Aufsatz in dieser Zeitschrift: „Das Einspruchs- und Nichtigkeitsverfahren nach dem geltenden Patentgesetz und die vorgeschlagene
Neuregelung," in der Nr. 18 vom 3./3. 1914, S. 127ff. darauf hingewiesen, daß die erschöpfende Prüfung von Erfindungen wohl erst durch die interessierte Technik selbst vorgenommen werden kann, und man kann wohl mit Fug und Recht behaupten, daß sich die amtliche Prüfung zu der von der Öffentlichkeit angestellten Prüfung im Einspruchsverfahren oft verhält wie Theorie und Praxis. Da Amerika ein Einspruchsverfahren überhaupt nicht und England ein solches nur in ganz beschränktem Umfange kennt, so kann die Tatsache der Patenterteilıng in diesen Ländern keineswegs als vollgültiger Beleg für die Patentfähigkeit angesehen werden.

Die Ausführungen von B a g l e y können deshalb kejne Veranlassung bilden, meinen Beitrag zur (Aeschichte der direkten Verfahren irgendwie zu berichtigen, zumal ich, wie ich schon früher betont und durch ausgiebige Hinweise belegt habe, mir das Material im wesentlichen aus der Literatur zusammengestellt habe. Bloß eines möchte ich hier noch hervorheben: Als man noch glaubte, mit einem wirklichen heißen Verfahren durchkommen zu können, wurde für das Verfahren Ko p pers der Ausdruck ,,halbdirektes Verfahren" zu dem ansdrücklichen Zwecke geprägt, damit eine angebliche Unvollkommenheit des Koppers'schen Verfahrens zu bezeichnen und ihm einen Makel anzuhängen. Jetzt, nachdem die Entwicklung mehr und mehr im Sinne dieser ,halbdirekten Verfahren" geht, kann nicht genug hervorgehoben werden, daß Koppers von vornherein diese jetzt allgemein anerkannte Arkeitsweise aufgegriffen hatte. Dieses "halbdirekteVerfahren"serfunden, seinem Werte nach erkannt und planmäßig ausgebildet zu haben, ist und bleibt zum wenigsten das unbestreitbare Verdienst von $K o p p e r s$.

Bochum, den 4./7. 1914

[A. 141.]

\section{Apparat zur Bestimmung von Kohlendioxyd nach Fresenius-Classen.}

\author{
Von A. Stchier.
}

(Eingeg. 11./7. 1914.)

Für die Bestimmung von Kohlendioxyd nach der Methode Fresenius-Classen hat sich noch folgender Apparat als bequem und zweckmäßig erwiesen. Er besteht, wie der gewöhnlich benutzte (siehe Lehrbuch von T r e a d w ell, Quantitative Analyse 1913, S. 322) aus dem Entwicklungsgefäß $E$, dem Kühler $K$ und den nachfolgenden Trockenund Absorptionsgefäßen $R, S$ und $T$. Jedoch wird er nicht, wie bisher üblich, aufgehängt, sondern die Gefäße stehen alle in kleinen (in der Zeichnung nicht angegebenen) Aluminiumfüßen, so daß die ganze Apparatur auf dem Arbeits platze aufgestellt werden kann, mit Ausnahme des Kühlers, welcher an einer Klammer befestigt wird. An beiden Enden wird jeweils eine mit konzentrierter Kalilauge beschickte Waschflasche ( $W 1$ und $W 2$ ) angebracht; $W 1$, damit nach Zersetzung des zu bestimmenden Carbonates ein kohlensäurefreier Luftstrom durch den Apparat gesaugt werden kann, W 2 dient als Schutzwaschflasche und ist mit einem Aspirator verbunden. Als Waschflaschen werden zweckmäßig sog. umschaltbare (von mir konstruierte) Flaschen verwendet, da ein Zurücksteigen der Kalilauge oft nicht $\mathrm{zu}$ vermeiden ist.

Der Kühler $K$ ist ein kleiner Kugelkühler von sehr starker Wirkung, da das Wasser durch die äußere der drei Kugeln zu- und durch die innere kleinste Kugel wieder abfließt, so daß der Wasser- resp. Säuredampf sich zwischen zwei dicken Schichten Kühlwasser befindet. Derselbe wurde von mir eigens zu diesem Zwecke abgeändert und hat in vorliegender Form nur einen Durchmesser von ca. $6 \mathrm{~cm}$. Wie sich Furch den Versuch ergeben hat, reicht er völlig aus, die entstehenden Dämpfe wieder zu kondensieren. $R$ vertritt die Stelle eines U-Rohres und wird mit Kupfervitriol-Bimsstein gefüllt. Die Waschflasche $S$ habe ich nach dem Muster des nachfolgenden Kaliapparates (nach $\mathbf{R} \mathbf{u} \mathbf{p}$ ) konstruiert. Sie wird mit konz. Schwefelsäure beschickt. Die in der Zeichnung angegebenen Pfeile deuten den Weg an, den das Kohlendioxyd nehmen muß, um nach $T$ zu ge- 
langen. Wie man sieht, wird das Gas in dieser kleinen Waschflasche im ganzen viermal gewaschen. Es genügt dies, um auch den letzten Rest an Wasser zu beseitigen. Der Apparat $T$ enthält konz. Kalilauge und stellt das Absorptionsgefä $B$ dar. Mit dieser neuen Apparatur wurde eine Reihe von Bestimmungen, welche gute Resultate ergaben,

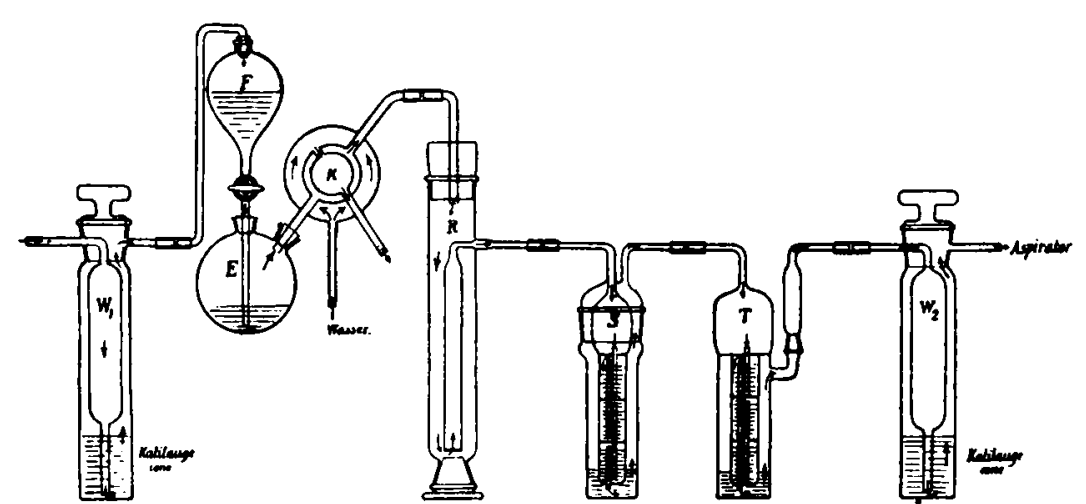

Thermometers versohmolzen wird, ist die Dauerhaftigkeit gewährleistet. Die Art und Wejse, wie die Verschmelzung erfolgt, soll gleichgültig bleiben.

Die Thermometer (D. R. G. M.) werden von der Firma Ed. Lucke, Fabrik wissenschaftlicher und technischer Instrumente aus Glas, Berlin N. 65, angefertigt und geliefert.

[A. 134.]

\section{Filtriertrichter. \\ Von J. F. Friedrichs. \\ (Eingeg. 29./4. 1914.)}

Büchnertrichter aus Porzellan haben den Nachteil, daß sie unterhalb der Siebplatte schwer zu reinigen sind, und daß infolge ihrer Undurchsichtigkeit leicht irgend welche Verunreinigungen unbemerkt bleiben können. Es lag daher ein Bedürfnis vor, Porzellan durch Glas zu ersetzen. Die Versuche, die in dieser Hinsicht gemacht worden sind, dürfen nur zum Teil als gelungen bezeichnet werden. Vor

ausgeführt. Sie hat vor allem den großen Vorzug der Standfestigkeit gegenüber dem älteren System. Ferner fällt das Füllen der Chlorcalcium- und Natronkalkröhren, sowie das dadurch bedingte Durchleiten eines Kohlendioxydstromes fort, da nur mit konz. Schwefelsäure getrocknet wird.

Der Apparat kann in dieser Form bezogen werden von der Firma Karl Kramer in Freiburg i. B.

Die Herstellung und den Vertrieb der Waschflasche, wie des Kugelkühlers hat die Firma Franz Hugershoff in Leipzig übernommen.

[A. 144.]

\section{Tragvorrichtung für Substanzenröhrchen an Schmelzpunkt-Bestimmungsthermometern.}

\author{
Von Georg Korher \\ Ingenleur in Bermsdorf bei Berlin. \\ (Eingeg. 20./6. 1914.)
}

Während man bisher die Tragvorrichtungen für Substanzröhrchen an die Thermometer kittete oder in anderer Weise an die Wandungen des Quecksilber-

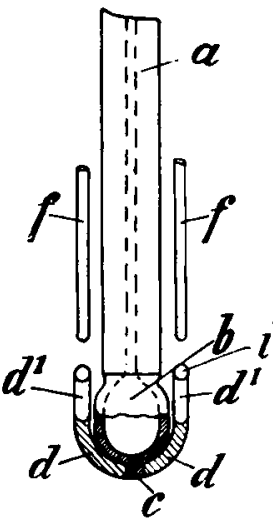
eies anliegend festklemmte, soll gemäß vorliegender Neuerung die Tragvorrichtung für die Substanzenröhrchen, gleichbleibend, ob als Gabel-, Kreuz- oder Ringrohr, mit dem Glasmaterial des Quecksilbereies in direkte Verbindung durch Verschmelzen oder dgl. gebracht werden. Durch diese Neuerung wird ein Abfallen und Zerbrechen oder Auslaufen der Substanzenröhrchen verhindert, und ein Abspringen der Tragvorrichtung vom Glasmaterial des Thermometers verhindert.

Auf der Zeichnung ist ein Ausführungsbeispiel in Fig. 1 in Ansicht mit teilweisem Längsschnitt und in Fig. 2 im Querschnitt dargestellt:

Das Glassteigerohr $a$ mit Quecksilberei $b$ des Thermometers zeigt im allge$d^{\prime} d^{\prime}$ meinen die bekannte Gestalt. An das Tragvorrichtung $d, d$ aus derselben Materie angeschmolzen. Diese Tragvorrichtung $d$ endigt oben in röhrenförmige Ausläufe $d^{1}, d^{1}$, in welche die Substanzenröhrchen $f f$ gestellt werden, um den Schmelzpunkt der betreffenden Substanzen zu bestimmen. Die Tragvorrichtung $d$ kann nun als gabeliges, kreuz- oder ringförmiges Rohr ausgebildet und massiv, hohl oder von beliebigem Querschnitte sein. Auch die Zahl und Ausbildung der oberen Ausläufe $d^{1}$ richtet sich nach dem jeweiligen Verwendungszwecke und kann verschiedenartigen Querschnittes sein.

Dadurch, daß die Tragvorrichtung gemäß vorliegender Erfindung ebenfalls aus Glas besteht, das mit dem Glase des einigen Jahren fertigte die Firma Greiner \& Friedrichs bereits Büchnertrichter aus Glas nach einem für die doppelwandigen Heißwassertrichter ausgearbeiteten Verfahren an. Da sie jedoch der Reinigung schwer zugänglich waren, den Anforderungen somit nicht voll genügten, und ihre Herstellung sich als zu kostspielig herausstellte, wurde von einer Publikation abgesehen. Eine wesentliche Verbesserung brachte die Modifikation, daß die Trichter nicht mehr wie früher aus einem Stück angefertigt wurden. Die beigegebenen Zeich-
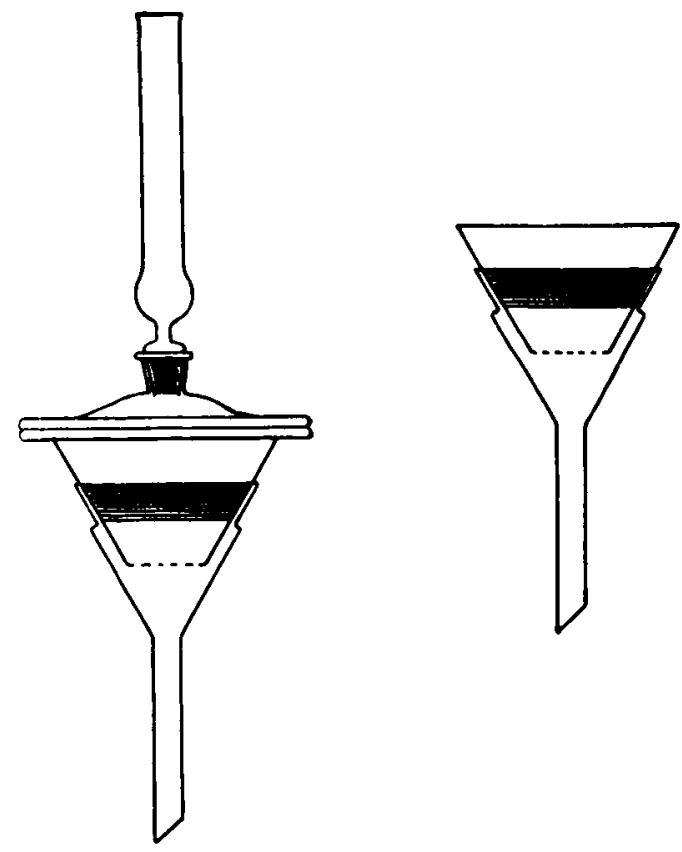

nungen machen eine eingehende Beschreibung überflüssig. In einem Trichter mit gebrochener Wand ist ein abgestumpfter Konus eingeschliffen, dessen Boden analog einem Goochtiegel siebartig durchlöchert ist. Da es diese Konstruktion erlaubt, auf den obersten Rand des Konus einen mit Chlorcalciumrohr versehenen Deckel aufzuschleifen, läßt sich der Trichter auch zum Absaugen von wasserempfindlichen Substanzen unter Ausschluß der Feuchtigkeit verwenden. Die Dimensionen gehen aus den Zeichnungen hervor, die die Trichter in halber natürlicher Größe abbilden.

Die Trichter werden von der Firma Greiner \& Friedrichs G. m. b. H., Stützerbach in Thüringen, angefertigt.

Chemisches Laboratorium der Universität Jena. [A. 81.] 